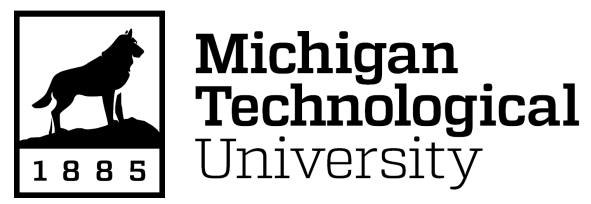

Michigan Technological University Digital Commons @ Michigan Tech

$1-1-1999$

\title{
Fluctuation properties of precipitation. Part IV: Finescale clustering of drops in variable rain
}

\author{
A. R. Jameson \\ RJH Scientific, Inc. \\ Alexander Kostinski \\ Michigan Technological University \\ A Kruger \\ University of lowa
}

Follow this and additional works at: https://digitalcommons.mtu.edu/physics-fp

Part of the Physics Commons

\section{Recommended Citation 10.1175/1520-0469(1999)056<0082:FPOPPI>2.0.C0;2 \\ Retrieved from: https://digitalcommons.mtu.edu/physics-fp/251 \\ Follow this and additional works at: https://digitalcommons.mtu.edu/physics-fp \\ Part of the Physics Commons}

Jameson, A. R., Kostinski, A., \& Kruger, A. (1999). Fluctuation properties of precipitation. Part IV: Finescale clustering of drops in variable rain. Journal of the Atmospheric Sciences, 56(1), 82-91. http://dx.doi.org/ 


\title{
Fluctuation Properties of Precipitation. Part IV: Finescale Clustering of Drops in Variable Rain
}

\author{
A. R. JAMESON \\ RJH Scientific, Inc., Alexandria, Virginia \\ A. B. Kostinski \\ Department of Physics, Michigan Technological University, Houghton, Michigan \\ A. KRUGER \\ Iowa Institute of Hydraulic Research, University of Iowa, Iowa City, Iowa
}

(Manuscript received 10 November 1997, in final form 9 March 1998)

\begin{abstract}
In recent studies it is shown that in variable rain the spatial distribution of drops is not Poissonian. However, these past studies were limited to 1-min drop counts, which likely correspond to spatial scales of a few hundred to several hundreds of meters.

In this work results based on 1-s drop counts using a video disdrometer are reported. It is shown that the clustering of raindrops previously found during intervals of $1 \mathrm{~min}$ also occurs during $1 \mathrm{~s}$ as well in convective rain. These latter temporal scales likely correspond to spatial features having dimensions from only a few to tens of meters. Combined with the authors' earlier results, these findings suggest that clustering of raindrops and meteorological variability span the range of scales from at least as small as a few meters to several hundreds of meters in convective precipitating systems. Consequently, non-Poissonian clustering reported in previous work (analyzing data accumulated over hours using 1-min drop counts) cannot be dismissed as artifacts in the data or errors in the processing. These studies appear to reflect accurately the true probabilistic character of rainfall.

Moreover, it is shown that the clustering is more prevalent and occurs over longer coherence times for larger than for smaller drops. An argument is given suggesting that the clustering of larger drops is likely associated with the larger scales of convection, whereas the clustering of smaller drops is likely more strongly influenced by smaller-scale turbulence. Furthermore, in convective rain it appears that the coherence times of drop size distributions will often be governed by the smaller drops. Using current technology, this will make it very difficult, at times, to adequately sample the larger drops in variable rain without mixing observations from more than one drop size distribution at the smaller sizes. Nevertheless, care must be taken since oversampling destroys information just as effectively as undersampling misses it.
\end{abstract}

\section{Introduction}

One of the many pleasures provided by a summer thunderstorm is the chance to observe curtains of rain weaving complex patterns aloft before splashing to their destiny like so many snakes slithering across the pavement. Although aesthetically pleasing, such patterns are the revealing expressions of both the complexity and the wide variety of scales that must be characteristics of rainfall in clouds.

In some sense this is not too surprising given the

Corresponding author address: Dr. Arthur R. Jameson, RJH Scientific, Inc., 5625 N. 32nd St., Arlington, VA 22207-1560.

E-mail: jameson@rjhsci.com stochastic nature of clouds (Jameson et al. 1998) and rain. Yet simply being random is not necessarily sufficient to explain all of the observed variability. In fact some stochastic processes actually tend to smooth out such fluctuations while others actually amplify them. Indeed it is often assumed, for example, that the number of raindrops of a given size striking an area in some time interval (i.e., the flux) can be described by Poisson statistics. Moreover, it is implicitly assumed, often simply because of necessity, that the fluxes at different drop sizes are also statistically independent.

Recently, however, it is shown that neither of these assumptions accurately reflects the true probabilistic nature of variable rain. That is, the spatial and temporal distributions of drops of one size often are not Poisson distributed because the fluxes at successive intervals and 
neighboring volumes are statistically correlated (Kostinski and Jameson 1997). Furthermore, the concept of size correlation is introduced. That is, the fluxes of drops having different sizes are also correlated so that they cannot be treated as independent random variables (Jameson and Kostinski 1998). As a result, convective, variable rain shows substantial, non-Poissonian fluctuations, whereas fluctuations in steadier, stratiform rain are more Poissonian (Kostinski and Jameson 1998).

Why? Because not only are the instantaneous fluxes themselves random variables, but so are their mean values (often referred to as the "meteorological variability"). This led Kostinski and Jameson (1997) to propose that the statistics of the flux of drops at a single size can be described by a doubly stochastic mixture of Poisson distributions each characterized by some mean value having a frequency of occurrence described by a probability density function (pdf) $f(\bar{k})$. The variance of such a distribution is then the sum of the "statistical" variance of a Poisson distribution having the observed "global" mean, $\mu$, and the "meteorological" variance associated with the pdf of the mean values contributing to $\mu$. Since rain consists of, say, $M$ different sizes of drops, these ideas naturally extend to the characterization of rain as a doubly stochastic Poisson process (Poisson mixture) of $M$ correlated random variables (fluxes) each having its own $f(\bar{k})$ and its own coherence time (Jameson and Kostinski 1998).

These conclusions, however, are based upon measurements using the Joss-Waldvogel disdrometer (Joss and Waldvogel 1967) that typically provides counts of drops at different sizes every minute. For a translation speed of a shower of say 5-20 $\mathrm{m} \mathrm{s}^{-1}$, this interval represents measurements over distances ranging from 300 to $1200 \mathrm{~m}$. It is natural to wonder, then, about what might be happening over shorter distances.

This interest is not merely academic, however. Shorter scales are important for understanding the effects of small-scale precipitation loading on cumulus dynamics, for understanding the onset of multiple scatter of microwaves passing through rain, and for understanding the effects of precipitation fluctuations on the signal statistics of all scanning remote sensing devices for measuring precipitation, from lasers to radiometers (Jameson and Kostinski 1996). Moreover, because measurements of drop counts on briefer intervals require different instruments, such observations act as an independent test of earlier concepts free from any idiosyncracies unique to the Joss-Waldvogel disdrometer (e.g., see Sheppard and Joe 1994).

The instrument used in this study is the University of Iowa, Iowa Institute of Hydraulic Research video disdrometer (see http://ias.tu-graz.ac.at/distro.html for further information). Briefly, two light sources generate orthogonal light sheets that are projected through narrow slits onto two line scan cameras-that is, horizontal, linear arrays of light sensitive detectors sampled on the order of $30 \mu$ s to yield a continuous data stream having no "dead" times. The optics are designed so that seen through the camera lens, the slits appear evenly and brightly lit. Particles falling through the beams of light appear as dark silhouettes against this background. The light sources and cameras form the sensor unit that is then exposed to the precipitation. Thus, the operation is similar to a flatbed scanner except that the hydrometeors move rather than the line-scan camera and light sheet. The effective sample area is approximately $10 \mathrm{~cm}$ $\times 10 \mathrm{~cm}$ or twice that of a Joss-Waldvogel disdrometer. The time series of particle images are then processed to yield the location (to within $0.2 \mathrm{~mm}$ horizontally), size, and other parameters describing the particles. This information is normally integrated from $15 \mathrm{~s}$ to hours to yield other quantities such as rainfall rate. However, for our purposes, we instead return to the original stored data and retrieve the recorded time of arrival to the nearest $\mathrm{ms}$ as well as the size of each drop to form a time series of the number of drops per second in bins 0.25 - $\mathrm{mm}$ wide in 0.25 steps from $0.625 \mathrm{~mm}$ up to whatever the largest size happens to be. Thus, it is possible to analyze the statistics of 1-s counts without the masking effects of "ringing" and dead times encountered when using the Joss-Waldvogel disdrometer (Sheppard and Joe 1994).

Using these measurements it is shown below that the characterization of rain derived in Parts I and II of this series of articles appears valid even down to the 1-s times. Moreover, drop clustering is apparent even on 1$\mathrm{s}$ scales associated with distances of a few to tens of meters suggesting that a more complete resolution of physical drop size distributions may require even greater temporal resolution over even larger areas.

\section{Drops of single size}

In this study, two brief convective rain events are presented, one lasting $201 \mathrm{~s} \mathrm{(} \sim 3 \mathrm{~min})$ and the other 773 $\mathrm{s}(\sim 13 \mathrm{~min})$. The shorter event is associated with a mean rain rate of about $13 \mathrm{~mm} \mathrm{~h}^{-1}$ with values exceeding 40 $\mathrm{mm} \mathrm{h}^{-1}$ for $40 \mathrm{~s}$ during the peak rain intensity, whereas during the longer rain, the average rate was $3 \mathrm{~mm} \mathrm{~h}^{-1}$ with values exceeding only $8 \mathrm{~mm} \mathrm{~h}^{-1}$ for $50 \mathrm{~s}$ during the maximum intensity. The time series of drop counts over a representative range of drop sizes are shown in Fig. 1. During the briefer shower (Fig. 1a) not only are features lasting less than $10 \mathrm{~s}$ evident, but there are several 1-s structures that appear simultaneously at several different drop sizes. Such short duration features are also evident during the longer rain event (Fig. 1b) as well. Thus, there are suggestions of correlated structure on scales approaching our temporal resolution, just as was found for the 1-min measurements using the Joss-Waldvogel disdrometer (Kostinski and Jameson 1997).

To explore this possibility, histograms of drop counts during these two periods are computed. In Part I recall that the histograms of drop counts using 900 one-minute 

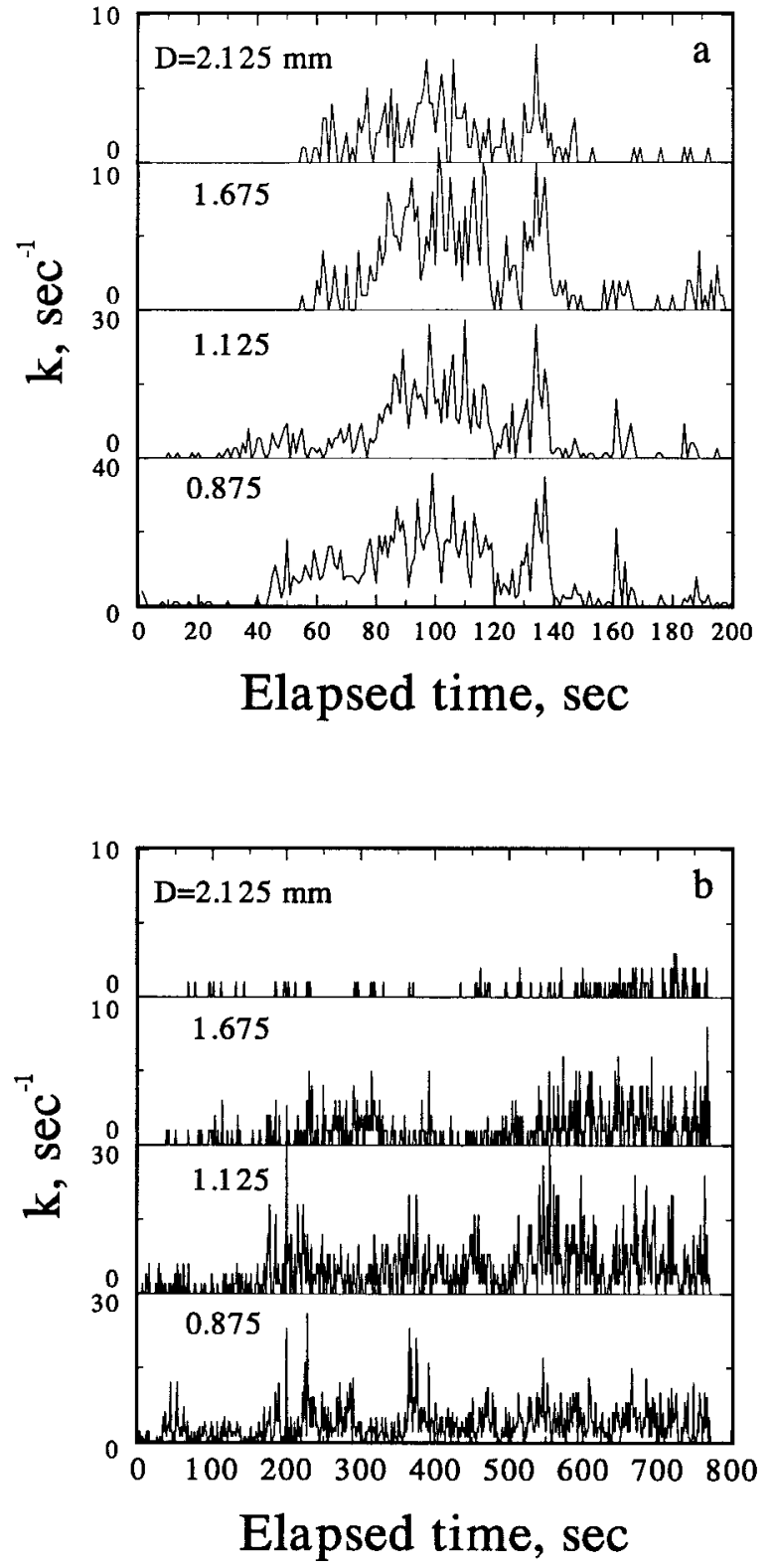

FIG. 1. Stacked time series of drop counts per second measured by the University of Iowa video disdrometer during the passage of (a) a brief 200-s convective shower for the indicated four different drop sizes and (b) a more extended rain event lasting $773 \mathrm{~s}$.

samples are often consistent with the geometric distribution given by

$$
P(k)=\frac{1}{1+\mu}\left(\frac{\mu}{1+\mu}\right)^{k},
$$

where $k$ and $\mu$ are the number and the global (longterm) mean number of drops per time interval, respectively. The beautiful feature of this distribution is that it involves only a single parameter, the mean value $(\mu)$, readily measured during any counting experiment.
As explained in Part I, (1) originates from the characterization of the counts of drops of one size as a Poisson probability mixture given by

$$
P(k)=\int_{0}^{\infty} P(k \mid \bar{k}) f(\bar{k}) d \mu=\int_{0}^{\infty} \frac{\bar{k}^{k} \exp (-\bar{k})}{k !} f(\bar{k}) d \bar{k}
$$

where the vertical bar denotes conditional probability and $\bar{k}$ is the mean corresponding to each patch of raindrops contributing to the mixture as described by the probability density function $f(\bar{k})$. This pdf may be considered to represent the distribution of "meteorological variability." When $f(\bar{k})$ is given by the exponential distribution

$$
f(\bar{k})=\frac{1}{\mu} \exp \left(-\frac{\bar{k}}{\mu}\right)
$$

Eq. (1) follows. Although it is perhaps not surprising that mixing as described by (2) is occurring when combining $900 \mathrm{~min}(15 \mathrm{~h})$ of data over widely varying meteorological conditions (Kostinski and Jameson 1997), we show next that (1) also describes observations over the much shorter time periods from 13 down to $3 \mathrm{~min}$.

Specifically, Figs. $2 \mathrm{a}$ and $2 \mathrm{~b}$ are the histograms, geometric fits, and Poisson pdf's (the latter two having the same mean value $\mu$ as measured) corresponding to the longer rain event for both the smallest drop size as well as for the largest drop size occurring at any reasonable frequency. It appears that over only $13 \mathrm{~min}$ (as opposed to $15 \mathrm{~h}$ in Part I), the histograms are well represented by a geometric distribution at the smaller and larger sizes although the Poisson pdf also appears adequate in Fig. 2b. Furthermore, Figs. 2c and 2d also show that a geometric distribution even describes the histograms over a 3-min interval. Thus, the results in Part I are not peculiar to having used measurements over $900 \mathrm{~min}$. Rather the meteorological variability observed previously over hours is actually also occurring even down to intervals of seconds.

To see this, the two-point autocorrelation function, $\eta(t)$, is computed at several different drop sizes for these two periods. Briefly, in Part I it was shown that

$$
\eta(t) \equiv \frac{\left[\overline{k(t) k(0)}-\mu^{2}\right]}{\mu^{2}}=\frac{\overline{k(t) k(0)}}{\mu^{2}}-1 .
$$

When $\eta(t)$ is nonzero, there is clustering and $P(k)$ deviates from the Poisson distribution. However, when $\eta(t)$ is zero, there is no clustering and Poisson statistics apply.

Figure 3 shows that over intervals of one to several seconds and at all sizes during both rain events, drop counts are correlated and, therefore, non-Poisson distributed. That is, even at $1 \mathrm{~s}$ as opposed to the 1-min temporal resolution in Part I, raindrop clustering and deviations from the Poisson distribution are still occurring in variable rain. Raindrop clustering, then, is not 

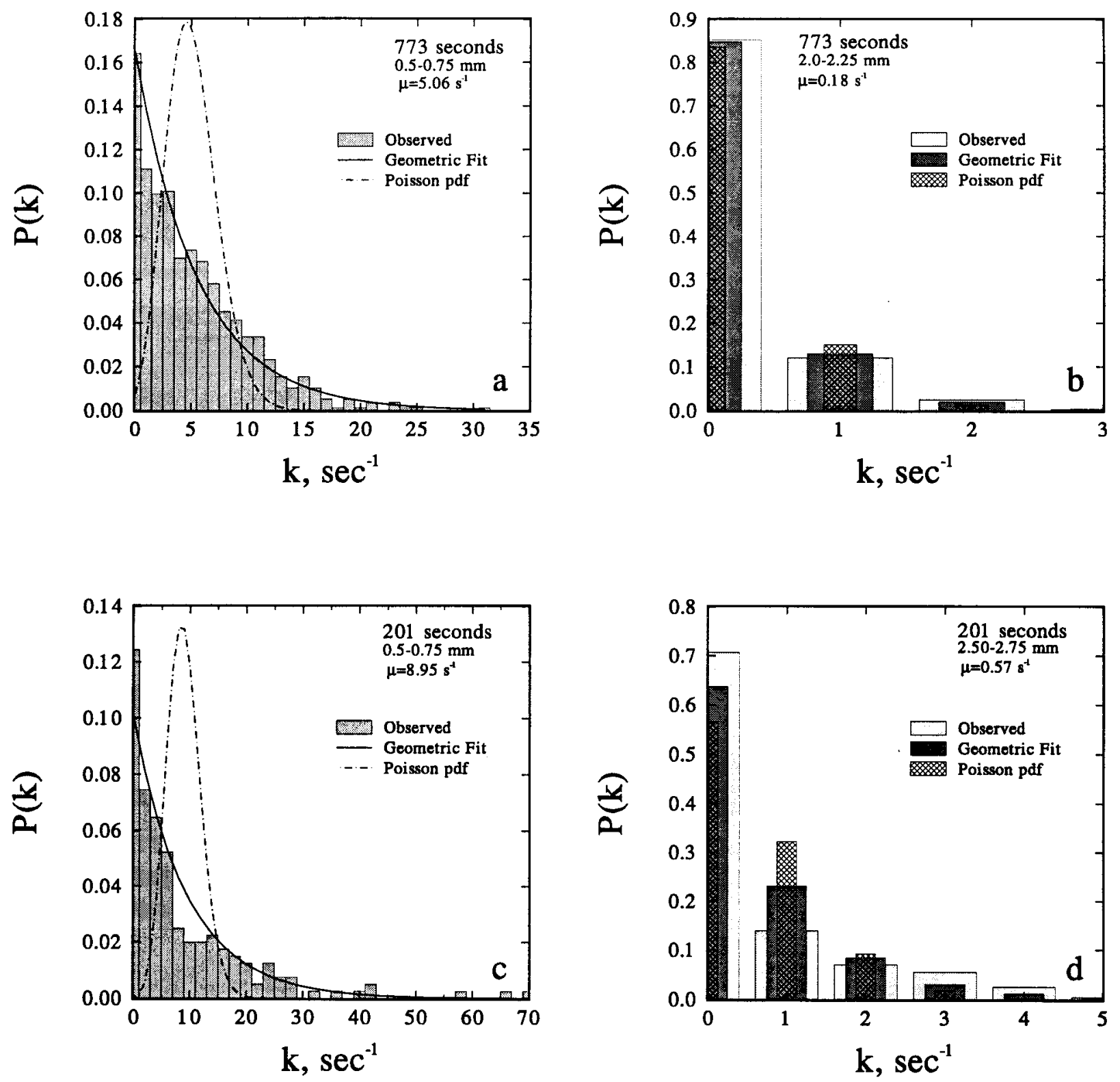

FIG. 2. Probability density functions $[P(k)]$ of observed drop counts per second $(k)$ for the longer rain event $[(a)$, (b)] and for the briefer rain event [(c), (d)] for the indicated size bins. Geometric fits and Poisson pdf's using the overall mean counts per second $(\mu)$ are plotted. Even during the brief rain, the histograms of the counts are well represented by the geometric distribution at small and large sizes although a Poisson pdf does almost as well at large sizes too because of the small number of drop counts.

just a phenomena over hundreds of meters (Part I); it even occurs over a few to tens of meters as well.

It is also apparent, however, that the statistical characters of the two rain events are different. Specifically, larger correlations appear during the 3-min rain suggesting that conditions are probably more variable then than during the 13-min rain. In particular, although the smallest drops in Fig. 3 are highly correlated for several seconds during the more intense 3-min rain, they are only weakly correlated for only a few seconds during the 13-min rain. Weaker correlations can be expected in so-called steadier rain (Kostinski and Jameson 1998). As previously pointed out in Parts I and II, sometimes drops of one size need not be clustered at all. That is, under less variable conditions, there can be interludes when a distribution of drops may be nearly Poisson over periods of hours. Nevertheless, in the more variable rain associated with convection (Kostinski and Jameson 1998), at least, it now appears that clustering occurs not just over distances of hundreds of meters but also over only a few to tens of meters as well.

Furthermore, in Parts I and II, it is shown that on 1- 

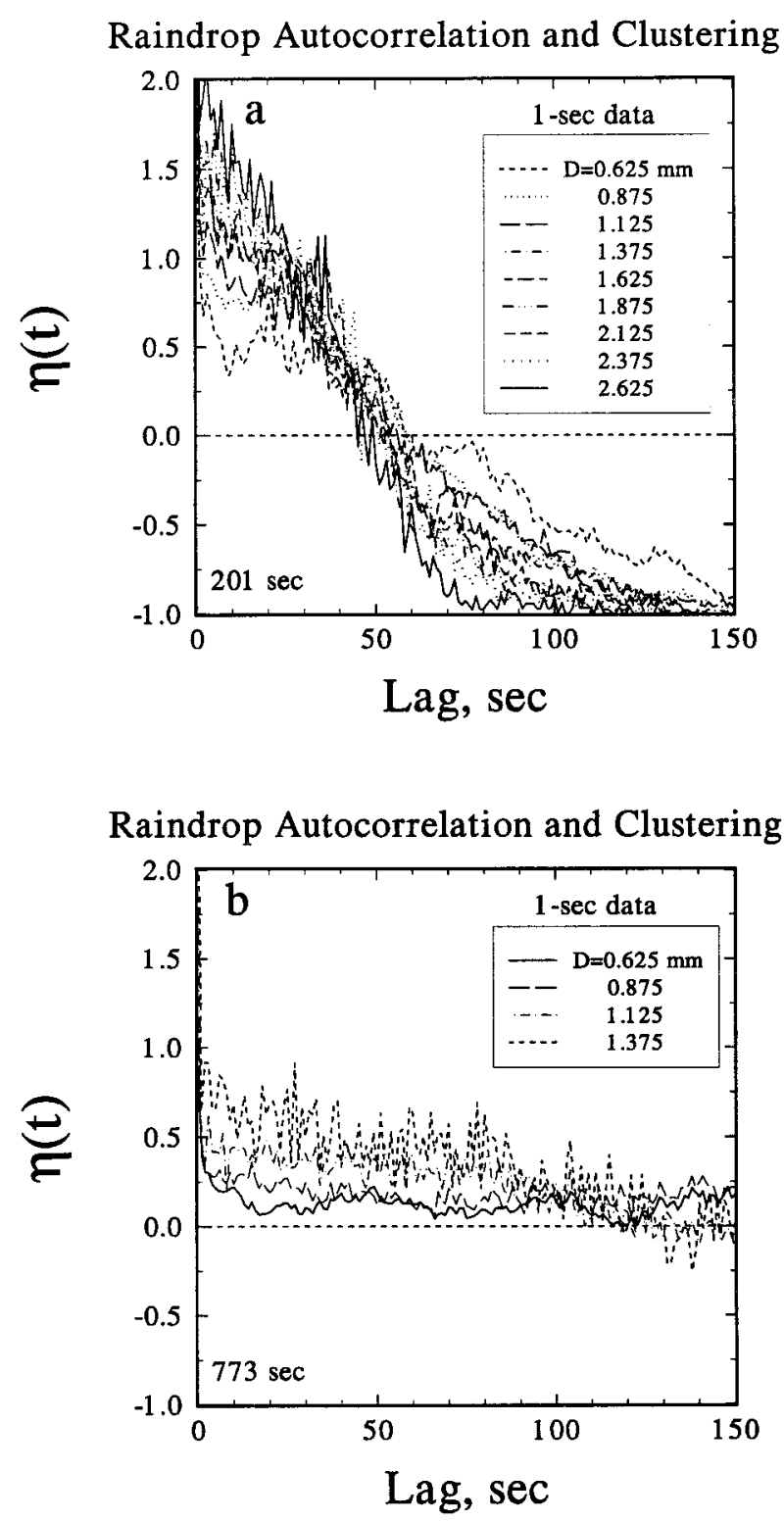

FIG. 3. The two-point autocorrelation function of drop counts per second for (a) the 201-s rain event and (b) for the 773-s rain event for the indicated sizes. The dashed line at zero indicates complete statistical independence as expected for a Poisson distribution. Note the enhanced clustering even down to the 1-s lag.

min timescales not just drop counts (concentrations) at one size are clustered (correlated), but that concentrations at different sizes are also correlated. This is confirmed in the next section even over intervals as brief as $1 \mathrm{~s}$. The implications with regard to the measurement of physical drop size distributions of interacting, correlated drops are then considered as well.

\section{Correlations among drops of different sizes}

In order to measure cross correlation of counts between drops of two different sizes, Jameson and Kos-
Interdrop Two-Point Cross Correlation
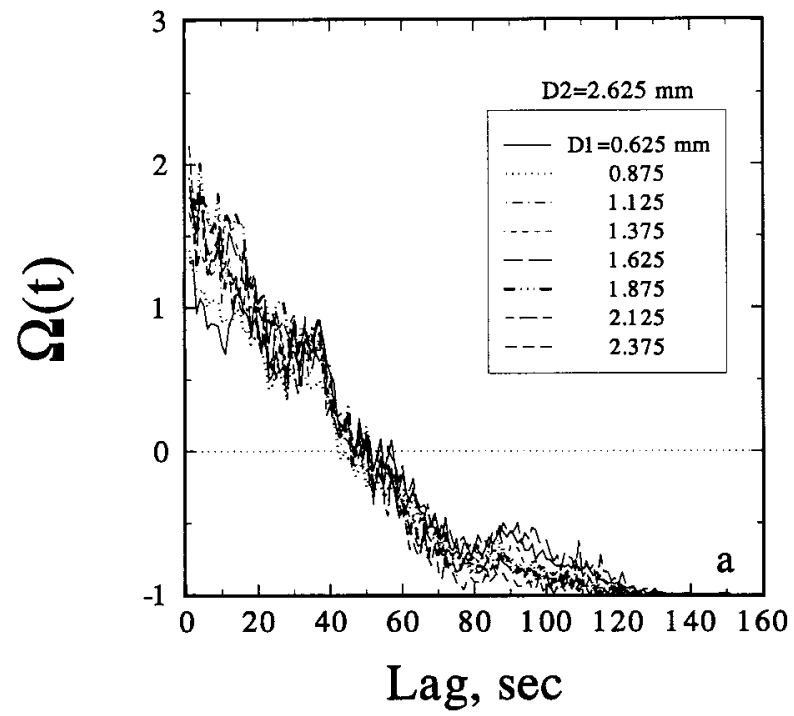

Interdrop Two-Point Cross Correlation

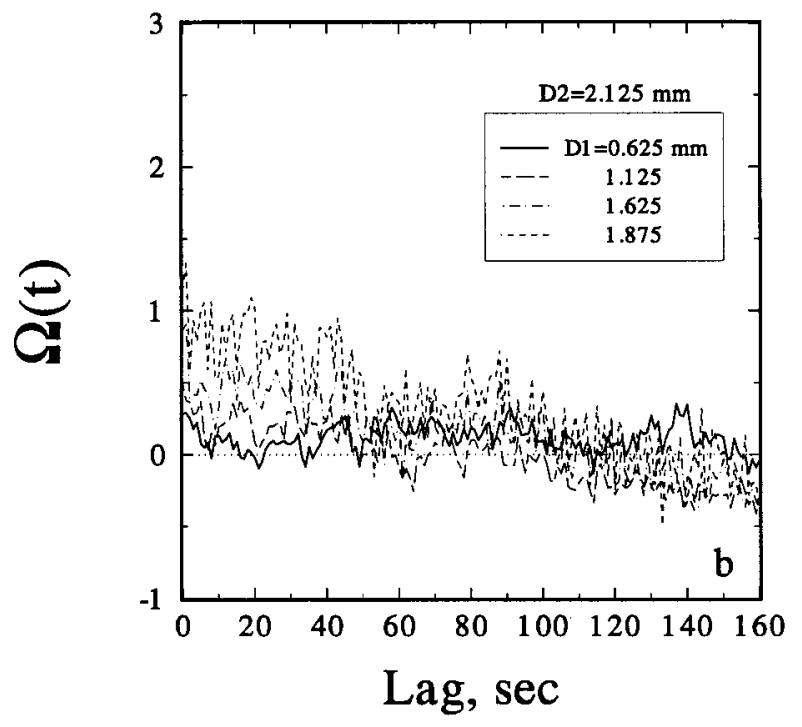

FIG. 4. The two-point cross correlation of raindrop counts between drops at the indicated diameters and (a) 2.625-mm size for the briefer rain and (b) $2.125 \mathrm{~mm}$ during the longer rain.

tinski (1998) modified (4) to become the two-point cross correlation given by

$$
\Omega(t) \equiv \frac{\left[\overline{k_{1}(0) k_{2}(t)}-\mu_{1} \mu_{2}\right]}{\mu_{1} \mu_{2}}=\frac{\overline{k_{1}(0) k_{2}(t)}}{\mu_{1} \mu_{2}}-1,
$$

where $k_{1}, \mu_{1}$ and $k_{2}, \mu_{2}$ correspond to two different drop sizes. Just as for $\eta(t), \Omega(t)$ is also zero if the two counts are statistically independent.

Figure 4 illustrates $\Omega(t)$ between one fixed drop size and other drop sizes for the two rain events. During the 201-s period (Fig. 4a), all the drop sizes appear to be 
correlated to varying degrees at least down to a rather well-defined coherence time of about $40 \mathrm{~s}$. This is consistent with results in Part II during the passage of a thunderstorm when the coherence time appears to be less than 1-2 min - that is, near the resolution limit of the Joss-Waldvogel disdrometer. Obviously, the nominal 1-min sampling interval of a Joss-Waldvogel disdrometer significantly exceeds the coherence time in Fig. 4a. It seems likely, then, that in many instances during convective rain, 1-min temporal resolution may be insufficient to resolve rainfall structure.

During the longer, 13-min rain event, however, the characteristics of $\Omega(t)$ are considerably different (Fig. 4b). Specifically, the cross correlation not only appears to be weaker, but the coherence times also are much more variable functions of drop size. For example, the smallest drops are hardly correlated at all with the largest size drops and then only briefly over periods lasting on the order of a few seconds. On the other hand, the larger drops not only appear more correlated, but the coherence times extend over several tens of seconds.

To illustrate this more clearly, we contour the twopoint cross-correlation matrix between all drop pairs corresponding to a time lag of $10 \mathrm{~s}$ for the two rain events (Fig. 5). Obviously, the correlations are larger during the 200-s rain event (Fig. 5a) than for the 770$\mathrm{s}$ rain (Fig. 5b). Because $\eta$ is a decreasing function of distance and time, Fig. 5 suggests that the spatial extent of rain patches is likely greater during the briefer rain. On the other hand, both plots show increasing correlation with increasing drop size.

In addition, though, we can form a matrix of coherence times for the cross correlations for all the different drop size pairs by noting the times when $\Omega(t)$ falls below, say, 0.3. These are plotted in Fig. 6. Although Fig. 5 shows greater clustering during the 3-min rain, Fig. 6 shows that for the larger drops the coherence times of this clustering were considerably briefer than during the 13-min rain. However, at smaller drop sizes the opposite is true-that is, smaller drops were associated with longer coherence times during the briefer as compared to the longer rain. During both rain events, however, larger drops exhibit longer coherence than do the smaller drops.

So what do these observations suggest? First, with regard to the characterization of the two rain events, it appears that the 3 -min rain is more clustered and variable than the 13-min rain. Moreover, the clusters are more coherent but of shorter duration like the waves of raindrops often seen splashing across the pavement during a thunderstorm. The 13-min rain, on the other hand, appears to consist perhaps of two superimposed components-namely, a "background" of nearly steady rain of smaller drops with clusters of larger drops passing through and lasting up to a minute or more.

In both events, though, the larger drops always show greater correlation of longer duration than do the smaller drops. (This same feature is also evident on 1-min time-
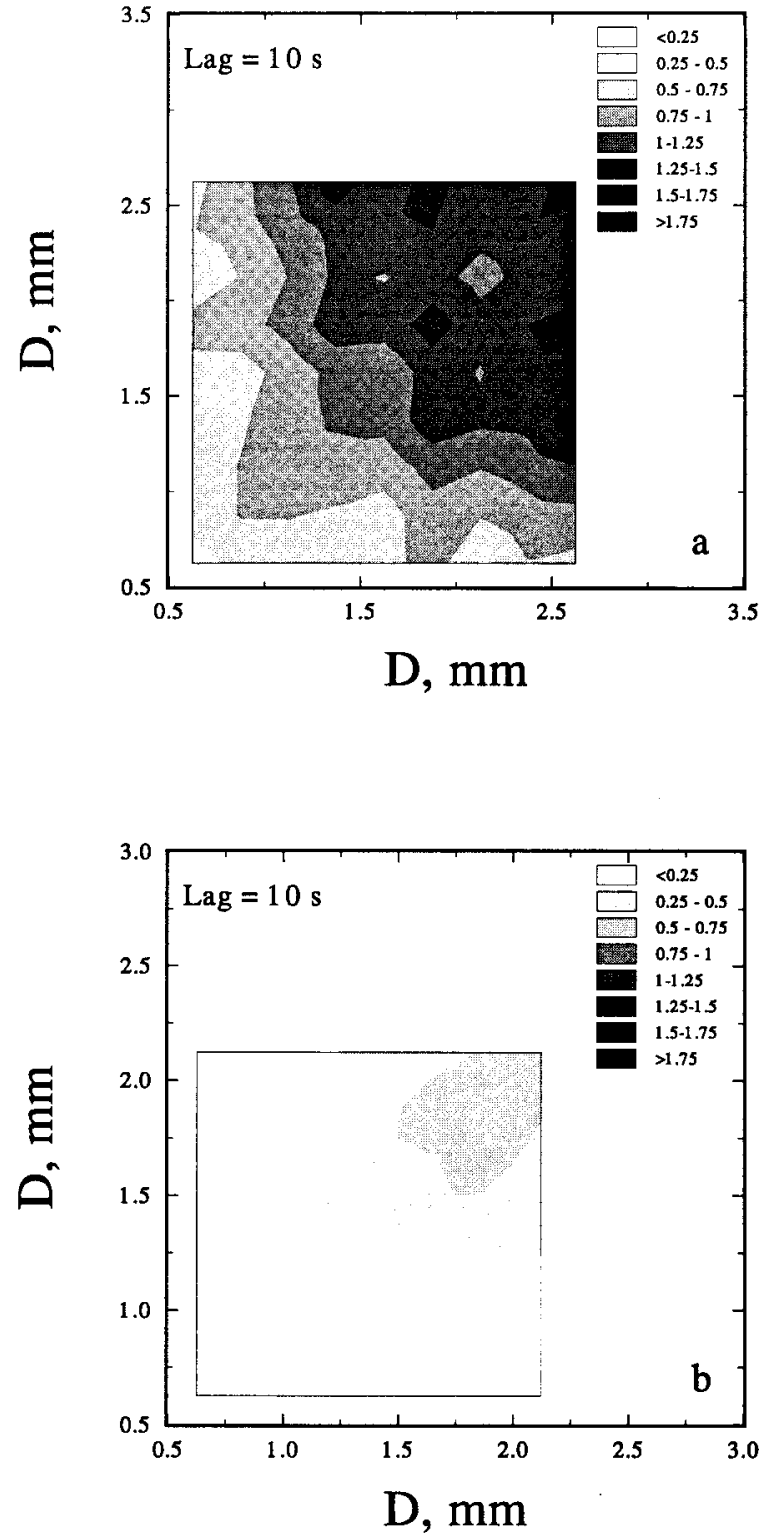

FIG. 5. Contour plots of the two-point correlation matrix for all drop size pairs for a time lag of $10 \mathrm{~s}$ during the (a) briefer and (b) longer rain events. Note the tendency toward greater correlations as drop sizes increase. The domain is restricted to those sizes having counts sufficient for meaningful correlation calculations.

scales in Fig. 3 in Part II.) Although we cannot yet say precisely why this should happen, one reasonable explanation might be that the larger drops are more closely associated with larger-scale convection. This should not be too surprising. Larger, more intense convection tends to be more moist (less entrained) and better able to keep drops aloft over longer periods so that they can grow to larger sizes. Furthermore, larger drops do not respond quickly to small-scale wind fluctuations (Beard and Jameson 1983). In contrast smaller drops are not only more easily created on many scales of convection, but 
they also respond more readily to smaller-scale wind fluctuations and turbulence. Thus, one might anticipate that smaller drops would tend to show reduced clustering of shorter duration, whereas the opposite would be true for the larger drops. Whatever the explanation, however, these differences suggest interesting implications with respect to the measurement of drop size distributions as discussed in the next section.

\section{On the measurement of drop size distributions}

In Part II it is argued that there are two types of drop size distributions. The first is the "physical" distribution of correlated concentrations among different sizes of drops resulting from the interactions among those drops. The second is an "average" distribution described by a mathematical expression representing the relation among uncorrelated concentrations at different sizes averaged over many different physical distributions.

In both cases, however, the drop size distribution is defined using mean fluxes at the different sizes observed over a measurement interval, $T$, consisting of many samples each collected during interval $t \ll T$. For physical drop size distributions it is envisioned that the mean concentration at each drop size remains constant during a coherence interval, $\tau$, during which the size distribution remains unchanged. We call this the drop size distribution coherence time. Moreover, when $t \ll T \ll$ $\tau$, then the distribution of counts remains Poisson at all sizes. In reality, however, often $T \gg \tau$. The distribution of drop counts is then no longer Poisson but something broader. When the meteorological variability [i.e., the variance of $f(\bar{k})$ ] is substantial, such as for an exponential distribution, then the distribution of counts may become very broad as illustrated by the geometric distribution (see Fig. 2). On the other hand, when the variability is less, the distribution of counts will be narrower and may often be well represented by a negative binomial distribution. [For greater elaboration see the discussion in section 2 and Fig. 12 in Part I. Also note that the last term in (10) in Part I should be $[\eta /(1+\eta))^{k}$.]

Ironically, because of the usual paucity of larger drops, measurement intervals are often selected in order to capture more of these drops for a "better" sample. Figure 6, however, suggests that this strategy may sometimes be in error because the coherence time of a physical drop size distribution ( $\tau$ above) is determined by the shortest coherence time of all the fluxes of all the different sizes contributing to the drop size distribution (Jameson and Kostinski 1998). This is frequently determined by the smaller and not the larger drops (Fig. 7 ). Thus, if $t \ll \tau \ll T$, the distribution of counts may indeed be more Poisson for those larger sizes (provided $T<\tau_{\mathrm{L}}$, where $\tau_{\mathrm{L}}$ is the coherence time of the larger drops). On the other hand, the distribution of counts may end up being very much broadened at smaller sizes because of mixing (i.e., $T \gg \tau_{\mathrm{S}}$, where $\tau_{\mathrm{S}}=\tau$ is the
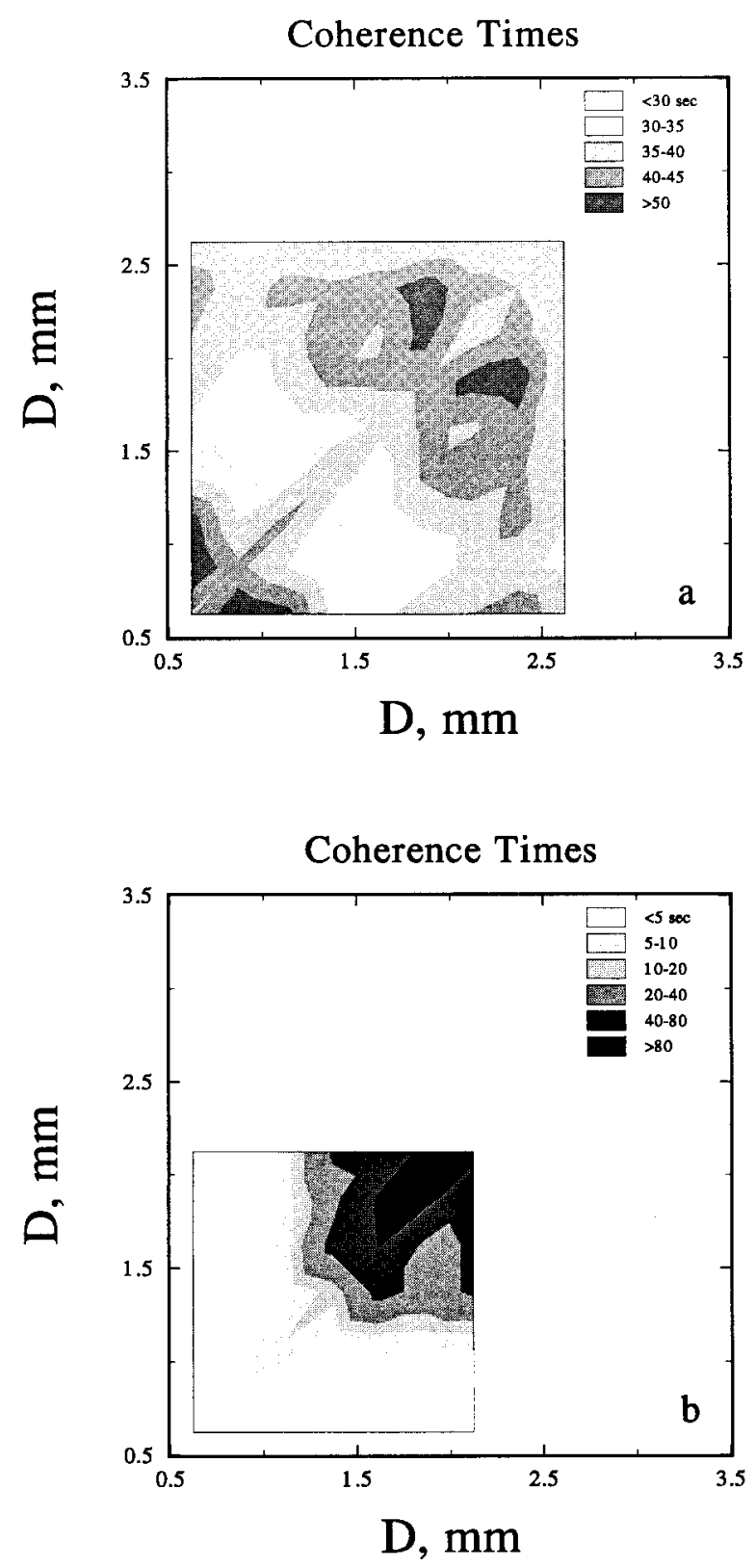

FIG. 6. Contour plots for all drop pairs of the matrix of coherence times required for the two-point cross-correlation function to fall below a threshold 0.3 . Note the tendency for longer coherence times as the drop sizes increase.

coherence time of the smaller drops). This is well illustrated in the next figure.

Using a coherence time of approximately $43 \mathrm{~s}$ (see Fig. 4), the histograms of drop counts for one period (87-129 s) during the 3-min rain are shown in Fig. 8. In addition, a negative binomial distribution is also fit to the observed histograms for three different drop sizes. Recall that the larger the shape parameter, $m$, for a negative binomial distribution, the closer it is to a Poisson 


\section{Autocorrelation Coherence Times}

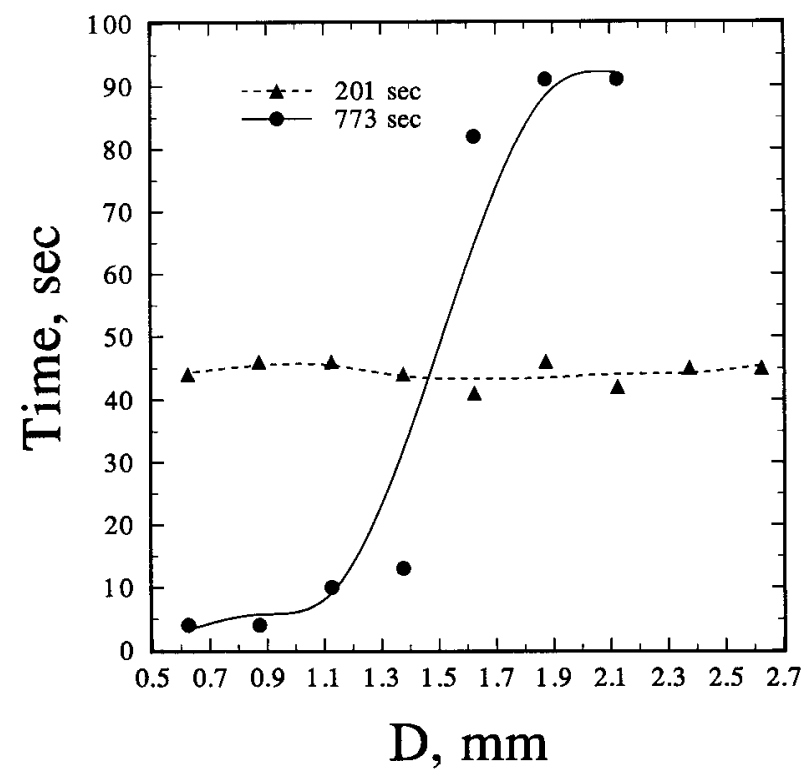

FIG. 7. The autocorrelation coherence times (Fig. 6) plotted as functions of drop diameter for the two rain events. The longer event shows much stronger dependence on drop diameter than does the shorter event consistent with Figs. 3 and 6.

distribution [see Kostinski and Jameson (1997) for elaboration].

During this interval, 181 drops are observed at the largest drop size, whereas 304 and 789 are counted at the successively smaller sizes, respectively. In spite of the much larger number of drops at the smallest size, it is indeed the distribution of the largest drop size that is closer to Poissonian in appearance. That is, the distribution at the smallest sizes is actually broader ( $m=$ $3)$ than is that at the largest size $(m=9)$ even though over four times as many drops are counted! Why? Because $t \ll \tau_{\mathrm{S}} \ll \tau_{\mathrm{L}}<T$ (Fig. 3a) so that there is more mixing,- that is, more different physical drop size distributions are contributing to the smaller sizes than to the larger size. However, in this example, $43 \mathrm{~s}$ still appears a bit too long (i.e., $T>\tau_{\mathrm{L}}$ ) since even at the largest size there is evidence of broadening of the histogram (Fig. 8c) beyond a pure Poisson distribution. Hence, it appears that even briefer measurement intervals (and, consequently, in order to achieve adequate sampling, shorter sample intervals over larger areas) may be required in order to avoid mixing. It is clear

FIG. 8. Histograms of the drop counts plotted as functions of the number of counts normalized by the mean value for a 43 -s time block during the brief rain event. Fits for negative binomial distributions are also plotted for the indicated shape parameter, $\mathrm{m}$. Plots are for small (a), medium (b), and large (c) drop size categories. Notice the broadening of the distribution as the size decreases even though the number of drops counted increases.
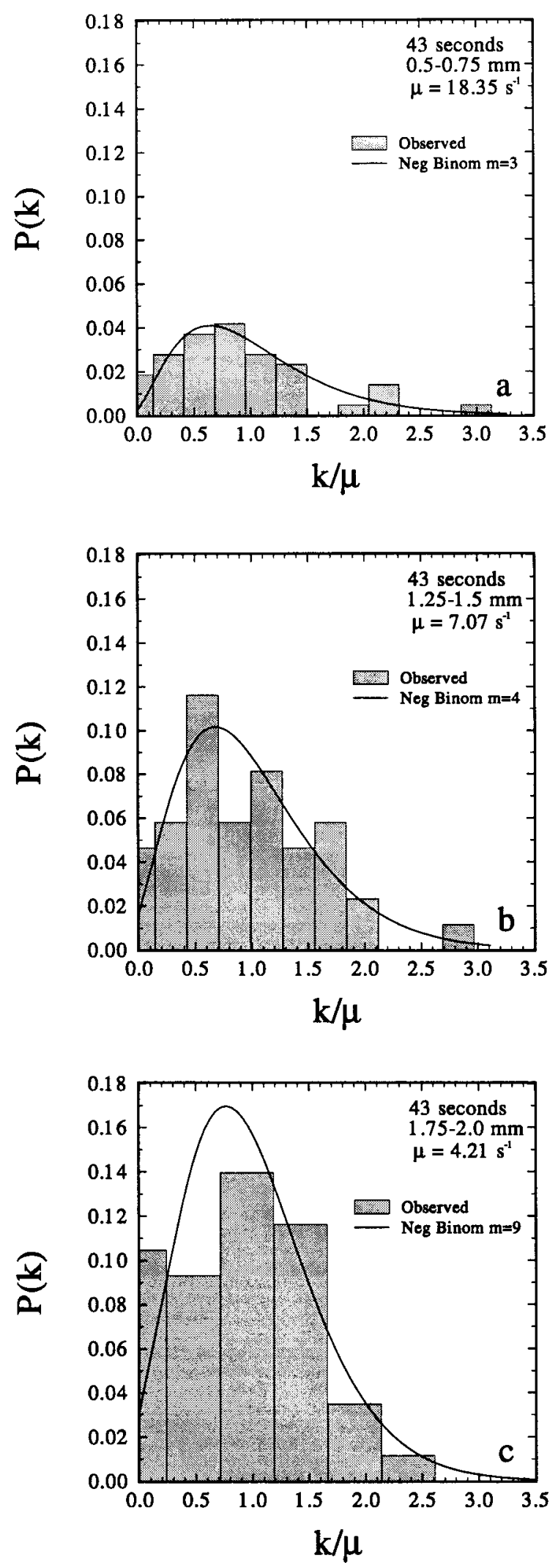


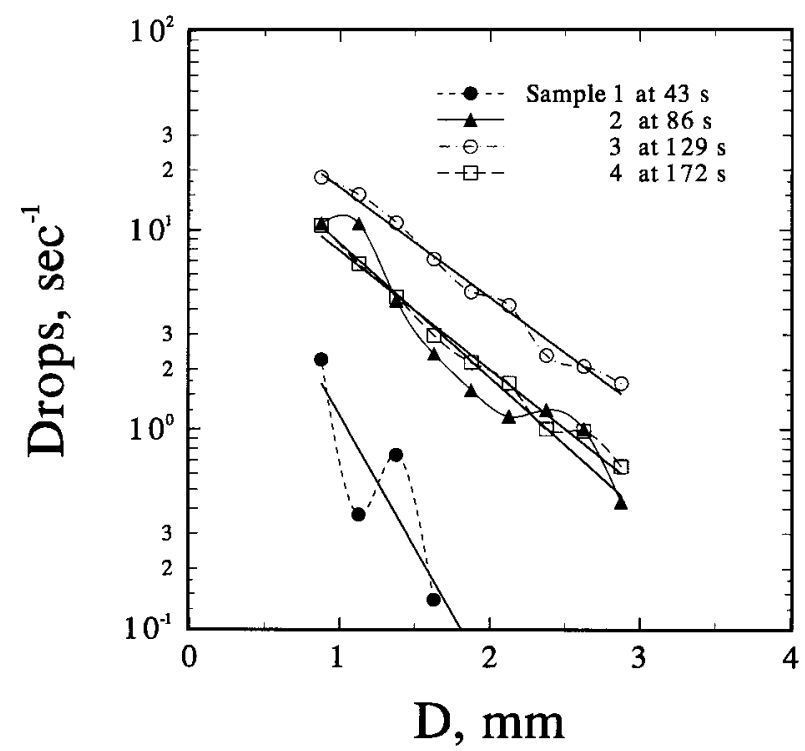

FIG. 9. Drop flux distributions for four consecutive time blocks (samples) each $43 \mathrm{~s}$ long during the brief rain event indicating the significant changes that can occur even during $3 \mathrm{~min}$. The solid lines correspond to exponential fits at each time block.

that 1-min intervals are totally inadequate for resolving the rapid changes occurring during the 3 -min rain in this example as well as in many convective rain events.

This can be seen by calculating a different distribution for each of the four successive 43-s time blocks during the 201-s rain as illustrated in Fig. 9. In this example, the solid lines represent exponential fits to the $43-\mathrm{s} \mathrm{av}-$ erage fluxes for each block. ${ }^{1}$ Obviously, even in a period as brief as 3 min there can be significant changes in the drop size distribution, changes that apparently at least can be partially resolved over $43 \mathrm{~s}$ using these 1-s measurements.

Although the variability around each exponential fit is reasonably small, the breadths of the distributions in Fig. 8 suggest that we have only partially separated the distributions. More complete resolution of the drop size distributions probably requires an even briefer measurement interval at even finer sample resolution over a larger sample area. After all, residual mixing still appears to occur in Fig. 8 even for a subminute, "fixed" 43-s coherence time. In fact, there is evidence that the coherence time itself changes even over only $201 \mathrm{~s}$.

To see this, for each of the four time blocks illustrated in Fig. 9 we compute the absolute magnitude of the deviation $\left(\left|n_{i}-\langle n\rangle_{i}\right|\right)$ of the observed average number

\footnotetext{
${ }^{1}$ While drop size distributions are normally computed for drop concentrations, we avoid uncertainties in the conversion from flux to concentration here by simply computing flux distributions directly. It is shown in Part III of this series (Kostinski and Jameson 1998) that the forms of the "flux" and "concentration" distributions are nearly identical. However, for our purposes here either distribution is sufficient.
}

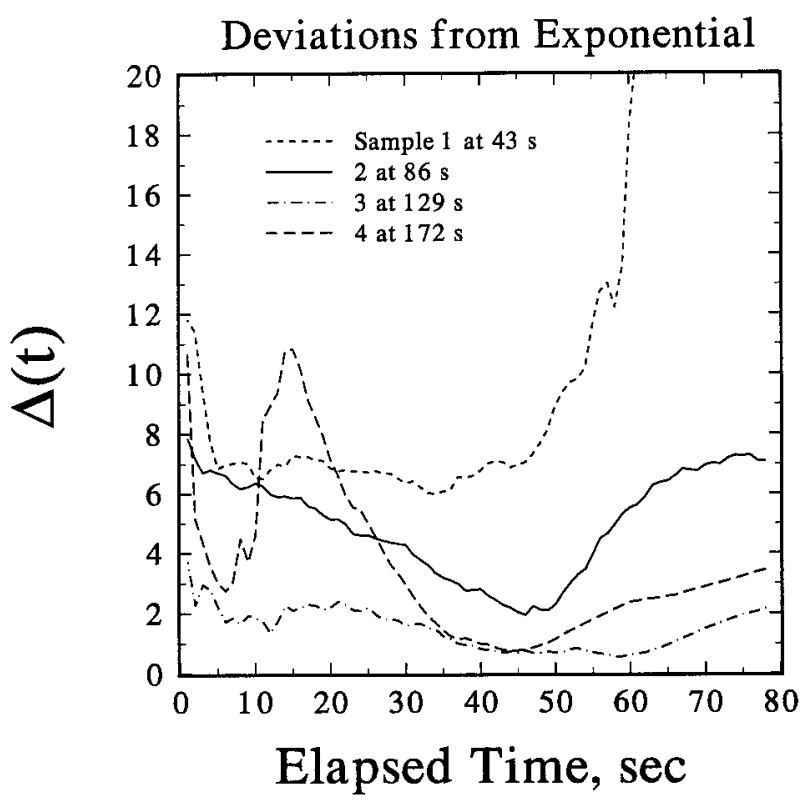

FIG. 10. Accumulated total fractional deviation from the exponential fits (Fig. 8) of flux averages as a function of measurement interval. Minima occur at different times ranging from 34 to $60 \mathrm{~s}$.

of counts, $n_{i}(t)$, from the average number expected for each exponential fit $\langle n\rangle_{i}$ at each drop size and for each measurement interval of length $t$. We then normalize each of these deviations by $\langle n\rangle_{i}$ (i.e., $\left|n_{i}(t)-\langle n\rangle_{i}\right| /\langle n\rangle_{i}$ ) and sum these quantities over all the drop sizes as functions of $t$ so that $\Delta(t)=\Sigma_{\mathrm{i}}\left(\left|n_{i}(t)-\langle n\rangle_{i}\right|\langle n\rangle_{i}\right.$. These accumulated fractional deviations, then, serve as overall measures of how closely the averaged observations match the exponential fit.

These are illustrated in Fig. 10. In all four cases there is a minimum in $\Delta(t)$, but these minima do not all occur at $43 \mathrm{~s}$. Rather in going from the first to fourth time block, they occur at 34, 46, 58, and 43 s, respectively, suggesting that the coherence time of the drop size distribution is likely changing as well.

Moreover, it is also clear that overaveraging produces increasing deviations from the exponential fit, whereas underaveraging also leads to greater deviations. This appears consistent with the notion that if the averaging period becomes too large, it exceeds the coherence time of the distribution leading to mixtures of physical distributions and increased uncertainties in the characterization of the drop size distributions themselves (Jameson and Kostinski 1998). Although previously shown to be true for sampling periods of several minutes in Part II, these data now indicate that the same is occurring even over times as small as tens of seconds in variable rain. Oversampling destroys information just as effectively as undersampling misses it.

\section{Summary and additional comments}

One of the significant findings of this work is that the results previously uncovered using 1-min samples 
from a Joss-Waldvogel disdrometer (Kostinski and Jameson 1997; Jameson and Kostinski 1998) over 15 h apply just as aptly to 1 -s measurements using a video disdrometer over tens of seconds. Consequently, the results reported in Parts I and II cannot be dismissed as artifacts in the data or errors in the processing. These studies appear to reflect accurately the true probabilistic character of rainfall.

Specifically, we show that at most drop sizes in variable rain the clustering of drops is occurring even down to 1 -s intervals likely corresponding to spatial scales of a few to tens of meters, a significant reduction from the few hundred to the several hundreds of meters reported in our previous studies. This is consistent with observations of "waves" or "sheets" of rain meandering across pavement during thunderstorms. Thus, fluctuating mean concentrations as described by $f(\bar{k})$ are pervasive in variable rain over many if not all scales.

Beyond simply reaffirming these previous results, however, the two-point correlation matrix is used here to show that larger drops are more strongly correlated over longer coherence times not only among drops of the same size but also among drops of different sizes than are the smaller drops. It is suggested that this occurs in part because the larger drops are likely associated with larger scales of convection, whereas the smaller drops are more strongly affected by smaller-scale turbulence.

In addition, the briefer coherence times of the smaller drops means that the coherence times of drop size distributions (Jameson and Kostinski 1998) is controlled not by the larger (as often assumed) but rather by the smaller drops. Therefore, the measurement of physical size distributions of interacting drops likely requires even briefer measurement intervals having finer sampling resolution (say on the order of $0.1 \mathrm{~s}$ ) over larger areas (on the order $1000 \mathrm{~cm}^{2}$ ) than used in this study for more satisfactory resolution of physical drop size distributions. This requires instrumentation not currently available. Yet it is these distributions that should be compared to results from numerical experiments that never attempt to include $f(\bar{k})$.

Finally, based on this study it appears that in variable rain, 1-min Joss-Waldvogel disdrometer samples will often be incapable of resolving physical drop size dis- tributions. Thus, up to now many reported measurements of drop size distributions in thunderstorms, for example, likely represent mixtures of several physical distributions. Although such mixing is not necessarily always a problem, it must be remembered that such distributions may never have existed as real physical entities but instead represent only some average condition. In subsequent simulations and calculations, then, it is important that the conditions of the simulations be consistent with the averaging used to derive the drop size distributions if the calculations are to yield physically meaningful results.

Acknowledgments. This work was supported by the National Science Foundation under Grants ATM9512685 (AK), ATM94-19523 (AJ), ATM97-08657 (AJ), and ATM97-12075 (AJ). Thanks are also due Prof. Krajewski for support of this collaboration and the Iowa Institute of Hydraulic Research for supporting Dr. Kruger in this research effort. We also gratefully acknowledge NSF Grant CMS95-00184 for funding the purchase of the video disdrometer, and Erin Rodichak for a careful reading of this manuscript.

\section{REFERENCES}

Beard, K. V., and A. R. Jameson, 1983: Raindrop canting. J. Atmos. Sci., 40, 455-462.

Jameson, A. R., and A. B. Kostinski, 1996: Non-Rayleigh signal statistics caused by relative motion during measurement. J. Appl. Meteor., 35, 1846-1859.

- and - 1998: Fluctuation properties of precipitation. Part II: Reconsideration of the meaning and measurement of raindrop size distributions. J. Atmos. Sci., 55, 283-294.

,-- , and R. A. Black 1998: The texture of clouds. J. Geophys. Res., 103, 6211-6220.

Joss, J., and A. Waldvogel, 1967: Ein Spektrograph für Niederschlagstropfen mit automatischer Auswertung. Pure Appl. Geophys., 68, 240-246.

Kostinski, A. B., and A. R. Jameson, 1997: Fluctuation properties of precipitation. Part I: On deviations of single-size drop counts from the Poisson distribution. J. Atmos. Sci., 54, 2174-2186.

- and — 1999: Fluctuation properties of precipitation. Part III: On the ubiquity and emergence of the exponential drop size spectra. J. Atmos. Sci., 56, 111-121.

Sheppard, B. E., and P. I. Joe, 1994: Comparison of raindrop size distribution measurements by a Joss-Waldvogel disdrometer, a PMS 2DG spectrometer, and a POSS Doppler radar. J. Atmos. Oceanic Technol., 11, 874-887. 